\title{
Antidepressants in Spine Surgery: A Systematic Review to Determine Benefits and Risks
}

\author{
Ahmed B. Bayoumi ${ }^{1,2}$, Oyku Ikizgul ${ }^{3}$, Ceren Nur Karaali ${ }^{3}$, \\ Selma Bozkurt ${ }^{4}$, Deniz Konya ${ }^{1}$, Zafer Orkun Toktas ${ }^{1}$ \\ ${ }^{1}$ Department of Neurosurgery, Medical Park Goztepe Hospital, Bahcesehir University School of Medicine, Istanbul, Turkey \\ ${ }^{2}$ Division of Neurosurgery, Department of Surgery, Hamilton General Hospital, McMaster University, Hamilton, ON, Canada \\ ${ }^{3}$ Bahcesehir University School of Medicine, Istanbul, Turkey \\ ${ }^{4}$ Department of Psychiatry, Medical Park Goztepe Hospital, Bahcesehir University School of Medicine, Istanbul, Turkey
}

\begin{abstract}
Antidepressant drugs can be advantageous in treating psychiatric and non-psychiatric illnesses, including spinal disorders. However, spine surgeons remain unfamiliar with the advantages and disadvantages of the use of antidepressant drugs as a part of the medical management of diseases of the spine. Our review article describes a systematic method using the PubMed/Medline database with a specific set of keywords to identify such benefits and drawbacks based on 17 original relevant articles published between January 2000 and February 2018; this provides the community of spine surgeons with available cumulative evidence contained within two tables illustrating both observational (10 studies; three cross-sectional, three case-control, and four cohort studies) and interventional (seven randomized clinical trials) studies. While tricyclic antidepressants (e.g., amitriptyline) and duloxetine can be effective in the treatment of neuropathic pain caused by root compression, venlafaxine may be more appropriate for patients with spinal cord injury presenting with depression and/or nociceptive pain. Despite the potential associated consequences of a prolonged hospital stay, higher cost, and controversial reports regarding the lowering of bone mineral density in the elderly, antidepressants may improve patient satisfaction and quality of life following surgery, and reduce postoperative pain and risk of delirium. The preoperative treatment of preexisting psychiatric diseases, such as anxiety and depression, can improve outcomes for patients with spinal cord injury-related disabilities; however, a preoperative platelet function assay is advocated prior to major spine surgical procedures to protect against significant intraoperative blood loss, as serotonergic antidepressants (e.g., selective serotonin reuptake inhibitors) and bupropion can increase the likelihood of bleeding intraoperatively due to drug-induced platelet dysfunction. This comprehensive review of this evolving topic can assist spine surgeons in better understanding the benefits and risks of antidepressant drugs to optimize outcomes and avoid potential hazards in a spine surgical setting.
\end{abstract}

Keywords: Spine surgery; Clinical outcomes; Antidepressive agents; Systematic review

\section{Introduction}

Although the main indications of antidepressants are psychiatric conditions, currently it is not uncommon for these drugs to be prescribed for non-psychiatric cases ow- ing to their proven benefits in a wide range of therapeutic fields. These indications include urinary stress incontinence, irritable bowel syndrome, premature ejaculation, and treatment or pain [1]. Surgical manipulation of subcutaneous tissues, bones, and ligaments may induce a

\footnotetext{
Received Sep 15, 2018; Revised Feb 18, 2019; Accepted Mar 14, 2019

Corresponding author: Ahmed B. Bayoumi

Department of Neurosurgery, Bahcesehir University, Tip Fakultesi, B2 Floor, Merdivenkoy Mahallesi, D 100 Caddesi, Kadikoy/Istanbul, Turkey

Tel: +90-538-264-78-57-25, Fax: +90-905-577-1424, E-mail: abayoumigcsrt@gmail.com
} 
significant postoperative pain [2]. The first notion of using antidepressants in neuropathy was based on the treatment of depression to relieve pain. In the 1960s, an autonomous pain-relieving activity was detailed for tricyclic antidepressants (TCAs). Currently, antidepressants are listed as a first-line treatment for neuropathic pain and they are used in several aspects of spinal surgery. The treatment of neuropathic pain, which involves several heterogeneous factors, is an important subject in relation to spinal surgery due to the relatively high prevalence and complex characteristics of the type of pain.

Pain is an important factor that can affect surgical outcomes and overall patient satisfaction following surgery. Depression is known to be a cause of altered pain perception; increased perception of pain can worsen symptoms of depression. Additionally, patients who suffer from chronic pain, such as spinal cord injury (SCI) or chronic lower back pain (LBP), are more prone to develop depression [3]. The incidence of pain is $65 \%-85 \%$ in patients with SCI, with half of these patients experiencing severe pain [4]. Major depressive disorder (MDD) is reported in $25 \%-30 \%$ of patients with SCI [5]. The mutual association between depression and pain results in a prolonged hospital stay and recovery time and poorer long-term outcomes [5]. Previous studies have shown that the treatment of preoperative depression correlated with reductions in pain perception and disability [6]. Furthermore, depression was found to be an independent predictor of postoperative delirium following surgery to the spine. It was concluded that postoperative delirium was a preventable cause of high cost and mortality rates in intractable cases relating to the spine [7].

Aside from the benefits of antidepressants in terms of clinical results, spine surgeons need to be aware of the potential hazards of using antidepressants. For example, an increase in intraoperative bleeding and allogenic blood transfusion is associated with serotonergic antidepressants. Platelet function assay evaluation and assessment of the risk for transfusion are recommended prior to major procedures in cases in which the patient is taking antidepressants prior to surgery. Following surgery, tapering off drug may be considered according to resolving depression and decreased levels of postoperative pain $[8,9]$.

A thorough understanding of the use of antidepressant drugs is required by surgeons in terms of their advantages and disadvantages. In the literature, the benefits and risks of using antidepressants in spinal surgery have been reported frequently in recent years. To the best of our knowledge, this is the first comprehensive review regarding the advantages and disadvantages of prescribing antidepressants in the context of spinal surgery. Our aim is to review the literature and provide surgeons with evidencebased data to improve decision making regarding antidepressant drugs that directly impact the clinical outcomes of patients undergoing spine surgery.

\section{Methods}

The PubMed/Medline, Scopus, Embase, and Central Cochrane Library were searched using a set of keywords in the form of the following code: "(antidepressant OR tca OR ssri) AND (spine OR spinal) AND (surgery).” All abstracts were double reviewed and checked by the authors according to the following preplanned list of inclusion criteria: (1) original clinical research relevant to the impact of antidepressant agents on the outcomes of spinal surgery or adverse events; (2) all antidepressant drugs were considered, including TCAs, selective serotonin reuptake inhibitors (SSRIs), serotonin and norepinephrine reuptake inhibitors (SNRIs), monoamine oxidase inhibitors, herbal drugs, or mirtazapine; (3) English abstracts; (4) at least 10 patients involved in the clinical study; and (5) articles published between January 2000 and February 2018. Our exclusion criteria were as follows: (1) clinical studies including < 10 patients; (2) review articles (secondary literature); (3) non-English full-text; and (4) basic science studies (preclinical studies and experimental bench work). The data are presented in two groups, interventional and observational studies, in Table 1 and Table 2, respectively. We reported for each article the last name of the first author, month and year of publication, aim of the study, relevant results and/or conclusion, type of epidemiological study, and level of evidence. A short narrative review was then supplemented to emphasize the advantages and disadvantages of the use of antidepressant drugs in the field of spine surgery based on the available evidence provided by previously published work in this area of interest.

\section{Results}

A total of 2,959 abstracts were identified following the use of the aforementioned set of keywords and following the removal of duplicates. As shown in Fig. 1, 17 articles were included in our review and are summarized in Tables 1 


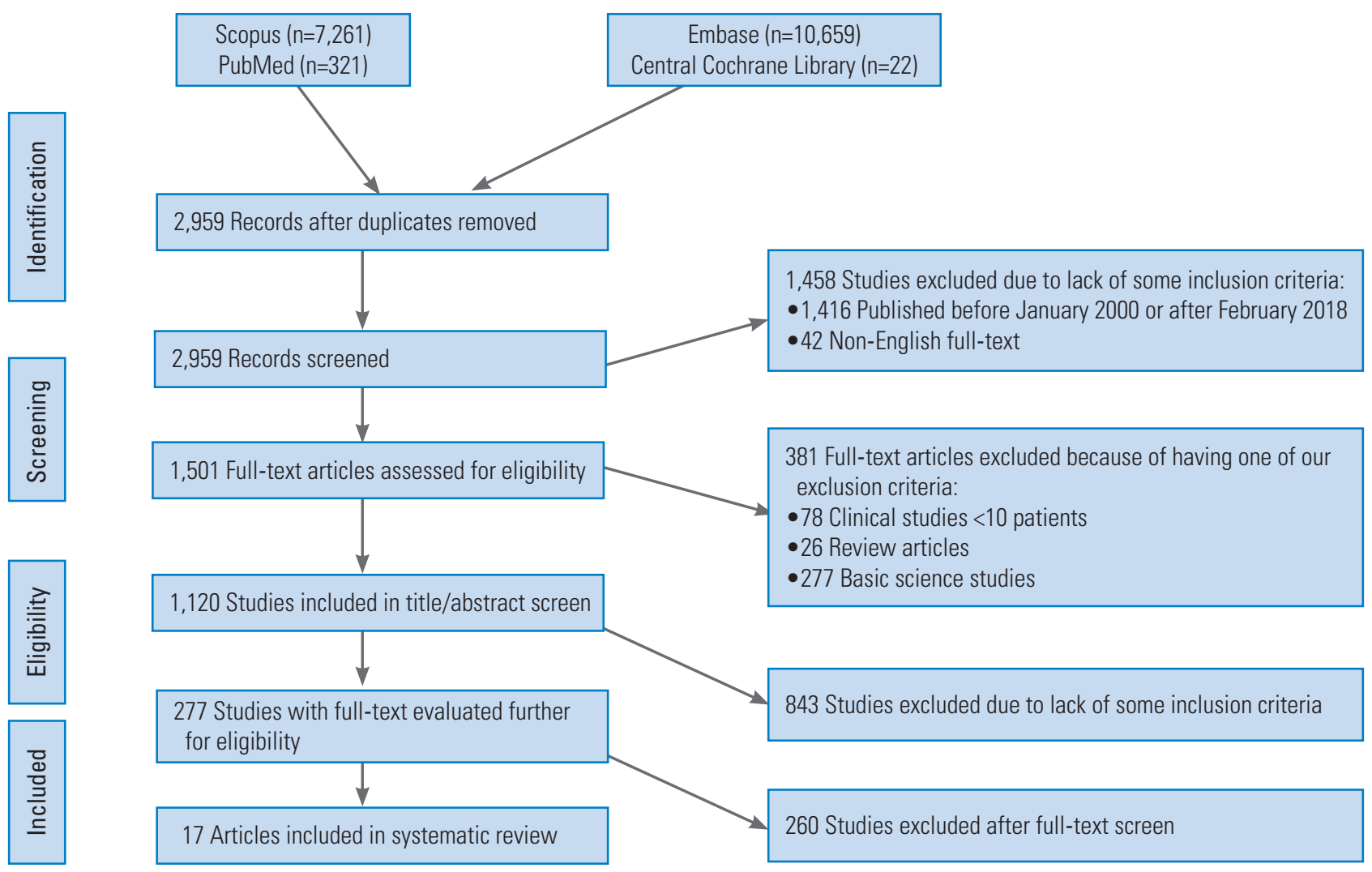

Fig. 1. Schematic diagram showing the PRISMA flow chart of our systematic review of the literature.

and 2 for interventional [4,10-16] and observational [69,17-22] studies, respectively. Only seven articles of the 17 (41.2\%) were interventional studies, all of which were in the form of randomized controlled trials (RCTs). The remaining 10 articles (58.8\%) were observational studies of different types (three cross-sectional, three case-control, and four cohort studies). A total of 53\% (9/17) were prospective studies, while $35.3 \%$ (8/17) were retrospective studies; $58.8 \%$ (10/17) reported favorable outcomes for antidepressant agents, whereas $37.5 \%$ (6/17) did not show a clear benefit; one article was based on a survey conducted in the initial steps of an RCT. TCAs were involved in four of the 17 studies, serotonergic drugs (SSRIs/SNRIs) were involved in nine of the 17 studies. Only 11.8\% (2/17) of the studies focused on cost. Of the 17 articles, three focused on the effect of antidepressants from a psychiatric perspective (to treat associated psychiatric comorbidity), while seven focused on antidepressant indication in the treatment of pain (without superadded depression or anxiety disorders). A total of five articles aimed to report major side effects (three for increased blood loss and two for possible osteoporosis). The median level of evidence provided by the included studies was 2.5 , whereas seven of the 17 articles (41.2\%) showed level I evidence in the interventional group. Of note, of the 17 articles, 13 articles were published between August 2015 and May 2017, whereas only three were published between November 2002 and January 2012.

\section{Discussion}

Antidepressant drugs are used in many conditions in spine surgery and may be highly efficient if the advantages and disadvantages are thoroughly scrutinized by clinicians. We conducted a survey in Turkey regarding the trends of antidepressant prescription in neurosurgical practice and the results indicated that many clinicians either do not consider using antidepressants or have insufficient knowledge in terms of the indications of the drugs. Among 336 neurosurgeons enrolled in the survey, of whom $36 \%$ were spine surgeons, $47 \%$ did not routinely prescribe antidepressants, and $22 \%$ of the non-prescribers considered antidepressants to have no role in neurosurgery. Of the spine surgeons, $46.3 \%$ did not prescribe antidepressants [23].

Although antidepressants can be a helpful tool to ad- 
dress challenging cases of spinal disease, there are conflicting opinions in terms of negotiating the risks that require clarification from the literature. These include the use of SSRI treatment in patients with osteoporosis, their effect on bone mineral density (BMD), intraoperative risk of bleeding and transfusion with serotonergic antidepressant use, pretreatment of depression for improving patient satisfaction, increased hospital costs, and longer hospital stays. In our experience, the tendency to prescribe antidepressants in tertiary medical care centers, such as research and university hospitals, is considered somewhat trivial among spine surgeons, as they have the ability to refer patients to psychiatrists if required. As a result of the ease of such referrals, many spine experts may omit the prescribing of antidepressants as an optional component in the conservative management of spine cases, which may lead to the masking of this useful group of invaluable drugs from practice. It is strongly encouraged that spine surgeons consider this area of pharmacotherapy to provide patients with additional benefits, provided they understand their potential adverse reactions. Evaluation of the risk/benefit ratio is the mainstay of clinical decision making. To the best of our knowledge, this is the first review on this topic that evaluates the literature in an evidencebased manner to guide clinicians while making decisions regarding the prescription of antidepressants for patients undergoing spine surgery or as a part of conservative treatment for non-surgical cases. Although evidence of bleeding tendency with serotonergic antidepressant agents appears solid in this review, evidence supporting the osteoporotic effect of antidepressants is clearly overstated.

The present study has limitations worthy of mention. First, only seven studies (of 17) were randomized clinical trials, whereas the remaining studies were observational (two prospective cohort, two retrospective cohort, four case-control, and two cross-sectional studies). Therefore, it is unlikely to address causality as the majority of the studies were observational. Measurement errors and selection bias were also potential limitations of the original articles, which may impact on interpretation. In crosssectional studies, no follow-up is expected. Additionally, the reliability of self-reported cognitive problems remain unclear compared with objective neuropsychologic assessment [24]. Three of the studies mentioned a small sample size as a limitation $[8,20,21]$. This review did not focus on a particular research question (i.e., testable null hypothesis) to run a meta-analysis for a specific scenario; instead, we reviewed all the relevant articles as each one, two, or maximum three articles had its own research question. Therefore, it would be either inefficient or invalid, in our opinion, to extract a net effect based on such scattered hypotheses. Running a meta-analysis requires homogeneity of all or most of the articles to detect the net effect, as well as its magnitude, direction, and statistical significance.

The future prospects, in this regard, aim toward conducting further RCTs comparing the efficacy of antidepressants as an adjunctive therapy to several drugs that are used more frequently in practice for neuropathic pain, namely pregabalin, gabapentin, or vitamin B6 (pyridoxine). It is anticipated that non-inferiority studies will offer a gateway for several antidepressant agents to expand their indications in cases of sciatica or failed back syndrome, before moving toward superiority trials. Another advance can be anticipated in establishing standardized conservative management protocols that emphasize the role of antidepressants, in order for spine surgeons to consider them among the plausible tools of pharmacotherapy, before declaring failure of conservative measures including medical treatment. Such strict protocols need to be adopted by international spine associations and committees, in the form of algorithms, to raise awareness of their profile and impact on patients that might be unclear for many surgeons. The financial burden of pushing for surgery in degenerative spine diseases may outweigh the cost of prescribing antidepressant agents in the right context.

\section{Literature Review}

\section{Neuropathic pain}

In the entire population, $>2 \%$ and $15 \%$ of the individuals of more than 40 years old suffer from neuropathy as a result of SCI, surgery, physical trauma to the nerve root, multiple sclerosis, or stroke. Several studies have been conducted to investigate treatment of neuropathic pain $[3,18]$.

In a randomized, double-blind, placebo-controlled phase III trial of 458 patients, $60 \mathrm{mg}$ duloxetine monotherapy for Japanese patients with chronic LBP was performed. By measuring the Brief Pain Inventory score, the duloxetine-treated group showed a 2-point improvement at week 14 ( $p=0.001)$. The study group also showed significant improvements in secondary measures, including Brief Pain Inventory, Patients Global Impression of 


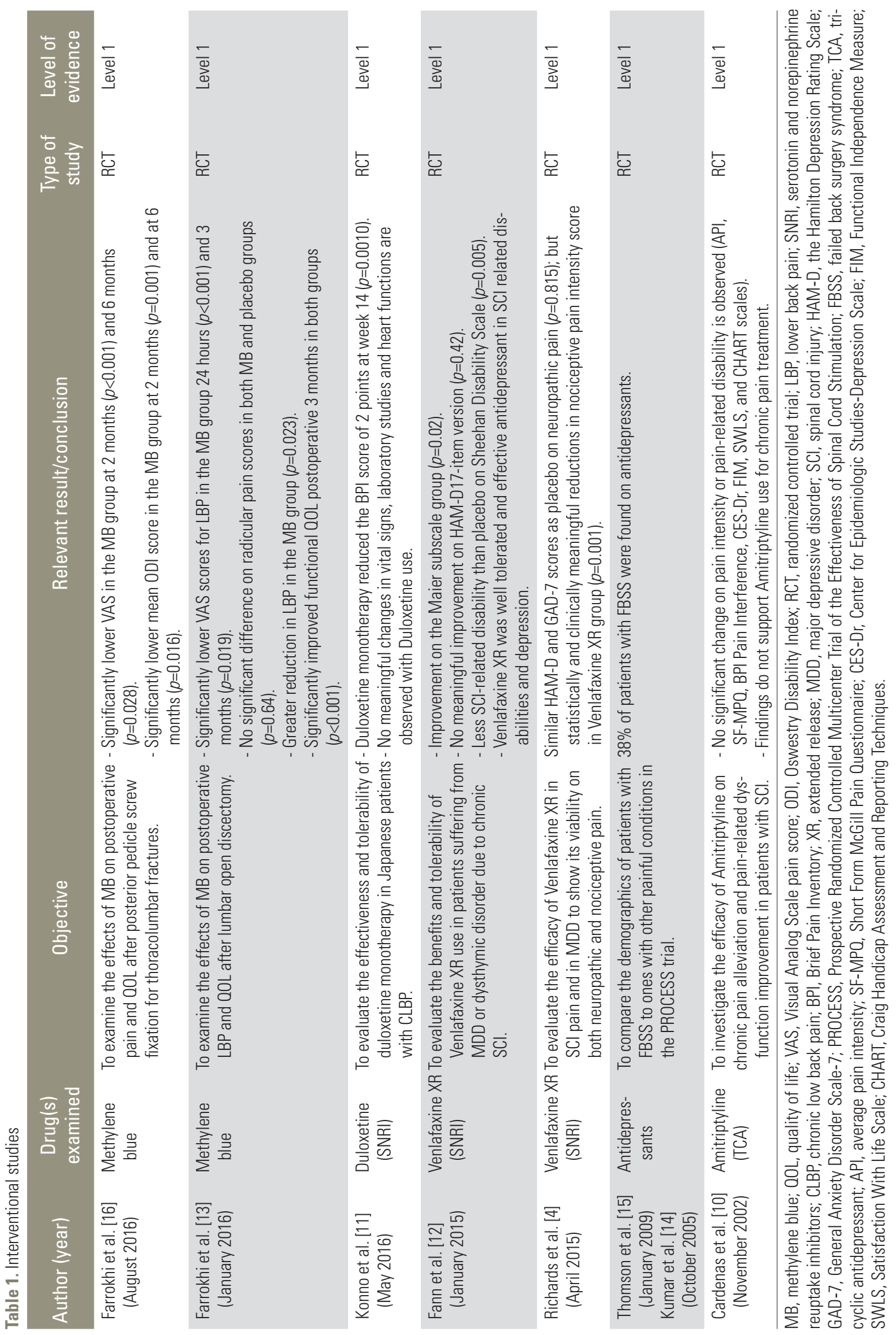




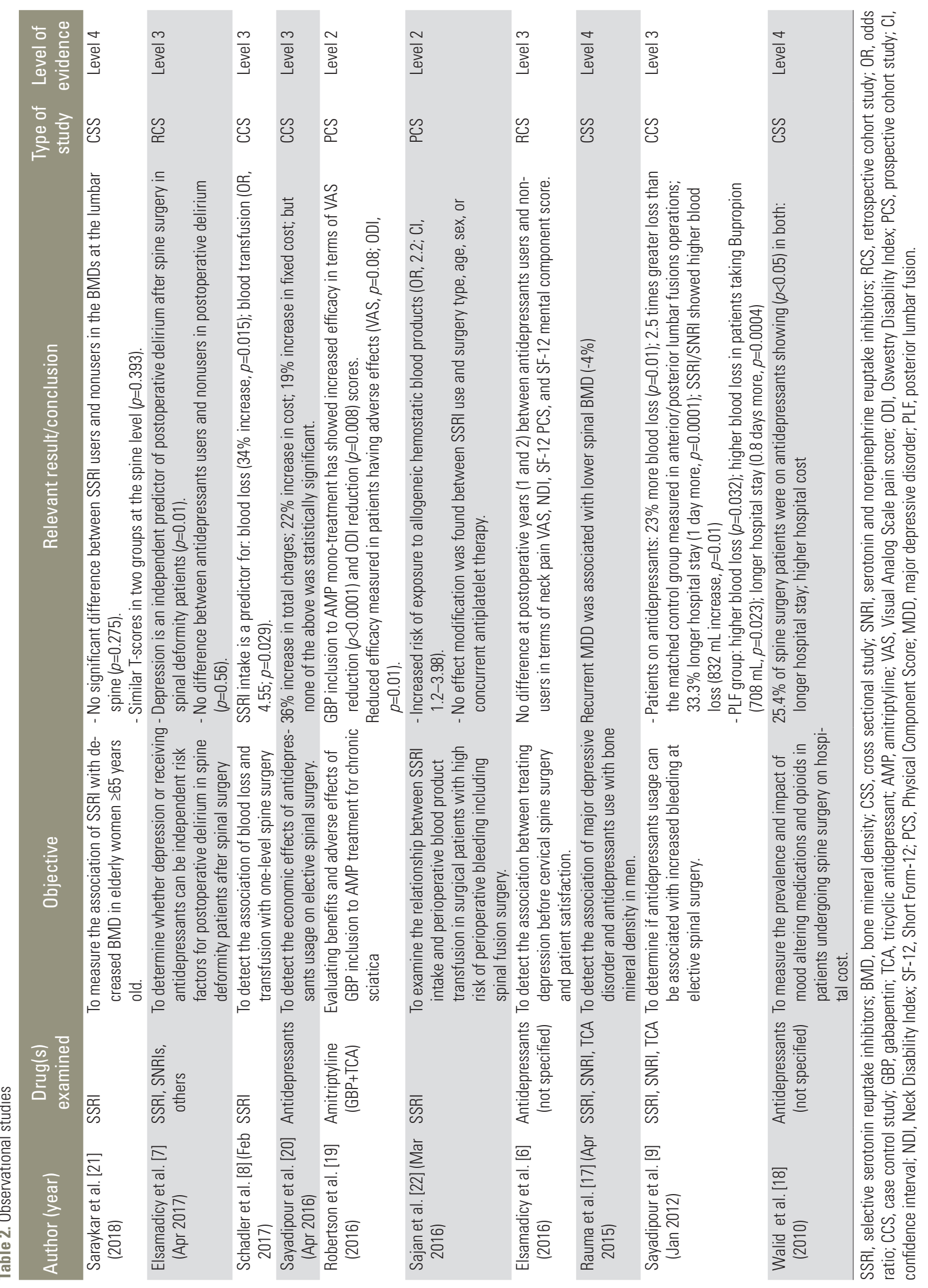


Improvement, Clinical Global Impression of severity, and Rolland-Morris Disability Questionnaire. Side effects, including somnolence, constipation, and nausea, were reported more frequently in the duloxetine group; however, they were not considered severe [11].

As TCAs are likely to cause several side effects, combination therapy to lower the required dose of a TCA drug by adding an additional drug for neuropathic pain is common in the management of chronic sciatica. One study investigated chronic management of sciatica in the form of prescribing an additional drug (gabapentin) for neuropathic pain with a dose of $300 \mathrm{mg}$ to $1.8 \mathrm{~g}$ to a preprescribed antidepressant drug amitriptyline $(10-50 \mathrm{mg}$ daily). In this prospective cohort study, which involved 77 patients, 56\% had reductions in the Visual Analog Scale (VAS) score $(p<0.0001)$ and Oswestry Disability Index (ODI) score $(p=0.008)$. A total of 82 side effects of 23 types, including amnesia, dizziness, confusion, fatigue, and ataxia, were reported in $53 \%$ of the patient population, whereas $34 \%$ withdrew from gabapentin in 1 week. Patients experiencing side effects benefited less from gabapentin in terms of reductions in VAS $(p=0.08)$ and particularly ODI ( $p=0.01$ ). Altered pain perception may have influenced these results [19].

It is reported that $65 \%-85 \%$ of patients with SCI experience pain [12]. TCA treatment for SCI-related neuropathic pain has been reported in the literature [25-27]. Furthermore, Richards et al. [4] evaluated venlafaxine extended release (XR) for SCI-related pain and treatment of MDD. No reduction in neuropathic pain $(p=0.815)$ was reported. Only patients suffering from nociceptive pain experienced a significant reduction $(p=0.001)$, whereas patients with mixed pain (nociceptive+neuropathic) experienced less reduction. The patients were evaluated at baseline and at 6 and 12 weeks [4]. In addition, the use of amitriptyline in SCI patients to improve pain and pain disability was reported as being ineffective by Cardenas et al. [10] in 2002 according to their RCT. Although amitriptyline is widely used for chronic pain problems, this study did not support its use in SCI patients. Patient satisfaction, according to Life Scale in that study, was significantly higher in the active placebo group $(p=0.004)$. Spasticity was more common in the amitriptyline group $(p=0.0005)$ [10].

\section{Patient satisfaction and long-term outcomes}

Non-specific pain, disability, poor quality of life (QOL), and prolonged hospital stay are important determinants of postoperative patient satisfaction $[6,7,13,16]$.

Farrokhi et al. [16] investigated pain and functional improvements with methylene blue (MB) injection on the soft tissue surrounding the fusion site following traumatic thoracolumbar fixation. Patients who underwent posterior pedicle screw fixation following thoracolumbar fractures received $1 \mathrm{~mL}$ of $\mathrm{MB}$ at a concentration of $0.5 \%$. Subsequent pain and QOL were compared with those in a control group treated with normal saline by applying VAS and ODI. In the MB injection patient group, the mean VAS scores were significantly lower compared with those in the control group at $2(p=0.001)$ and $6(p=0.028)$ months postsurgery. The ODI score was higher at the $2-(p=0.001)$ and 6 -month $(p=0.016)$ interval follow-ups. That indicated improved functional QOL. Although a few patients admitted using additional analgesic drugs, a single dose of MB injection was considered adequate to render the pain bearable following surgery, and QOL showed notable improvement [16]. Furthermore, Farrokhi et al. [13] investigated the effects of postoperative MB injection on LBP and functional outcomes following lumbar open discectomy in 115 patients. Outcomes in the MB-injected group were evaluated and compared with those in the saline-treated group. At the 24-hour and 3-month follow-ups, the mean VAS scores for the MB group were significantly lower $(p<0.001$ and $p=0.019$, respectively). LBP improvement was more marked in the MB group ( $p=0.023 ; 95 \%$ confidence interval, -1.37 to -0.10 ), whereas radicular pain did not differ $(p=0.64)$. Functional QOL improvement was noted for both groups $(p<0.001) 3$ months following the procedure [13]. It was concluded that intradermal MB injection was an easy, quick, and effective procedure to reduce postoperational problems including LBP, radicular pain, and functional QOL. Intrathecal injection should be discarded to avoid neurological deficits $[13,16]$.

Depression is considered to be an aggravating factor for intractable pain. A retrospective study including 140 patients examined the outcomes of depression treatment prior to cervical spine surgery. Patients who underwent anterior cervical discectomy and fusion (ACDF) were evaluated using the Neck Disability Index (NDI) for functionality, Short Form-12 (SF-12) for mental health, and VAS for neck pain, prior to surgery and 3, 6, 12, and 24 months following surgery. None of the measures showed a significant difference between the patient group pretreated with antidepressants and the non-depressed control group 
( $p=0.11$ for NDI; $p=0.63$ for SF-12; $p=0.80$ for VAS). These results suggest that pretreatment eliminates the pain-altering effect of depression and improves clinical outcomes with higher patient satisfaction [6].

In addition to increased pain and postoperative disability rates, depression is considered to be an independent risk factor for postoperative delirium. In a retrospective study of 923 adult patients with spine deformity, the delirium rate was 2 -fold higher for depressed patients $(10.59 \%$ versus $5.84 \%, p=0.01$ ). Strong correlations between postoperative delirium and poor surgical outcomes, including high complication rates, prolonged hospital stay, increased health cost, and in-hospital mortality, have been reported [7]. In a retrospective study, the outcomes 6 months after surgery in pretreated depression patients and nondepressed patients were reported to be similar [6]. Overall, depression is a preventable cause of pain, pain-related disability, and postoperative delirium that leads to inferior outcomes for surgery. Therefore, pretreatment with antidepressants may be considered for favorable postoperative outcomes in terms of pain and delirium $[6,7,13,16]$.

\section{Intraoperative bleeding and perioperative transfusion risk}

Several studies have shown an increased risk for intraoperative bleeding with perioperative serotonergic antidepressant treatment $[8,9,22,28,29]$. SSRIs induce serotonin depletion in platelets with reuptake inhibition, leading to impaired aggregation and vasoconstriction $[8,9,22]$.

In a study performed by Sayadipour et al. [9] in 2012, intraoperative blood loss was reported for the study group of patients taking antidepressants compared with those not taking (control group), who underwent ACDF, posterior cervical fusion, combined anterior-posterior cervical fusion, anterior lumbar interbody fusion, posterior lumbar fusion (PLF), or combined anterior-PLF. The results indicated that the mean blood loss was $23 \%$ higher in those taking antidepressants than in the control group ( 298 versus $241 \mathrm{~mL}, p=0.01$ ). The blood loss was profound in anterior-PLF surgeries with 2.5-fold higher rates than in the matched group. Among the antidepressants, SSRI/ SNRI drugs led to more marked bleeding (334 versus 241 $\mathrm{mL}, p=0.015)$. According to the surgery type, the blood loss difference in PLF patients between the study and control groups was more than in other subgroups (560 versus $457 \mathrm{~mL}, p=0.032$ ). Patients prescribed bupropion who underwent PLF showed higher blood loss than the control group (708 versus $457 \mathrm{~mL}, p=0.023$ ) [9]. This evidence was corroborated by Schadler [8] in 2017, who reported a $34 \%$ increase in the blood loss with SSRI intake in patients who underwent single-level fusion surgery of the lumbar spine $(p=0.015)$. However, SNRIs have not been proven to be significantly related to prediction of blood loss due to their low affinity to serotonin transporters $(p=0.375)$. Female gender was also a strong indicator for the risk of blood loss (odds ratio [OR], 5.952; $p=0.029$ ). In addition, SSRIs have been associated with perioperative allogeneic blood transfusion (OR, $4.55 ; p=0.029$ ) [8].

Similar results were reported by Sajan et al. [22] in 2016. Preoperative SSRI administration increased the probability of hemostatic transfusion by 2 -fold, whereas SNRIs and other antidepressants were statistically insignificant. However, SNRIs had similar trends to those of SSRIs [22]. Switching patients to antidepressants that do not work via serotonin reuptake inhibition may be considered prior to a major surgical procedure. A Platelet Function Assay (PFA) is the only effective test for risk assessment due to serotonin reuptake inhibitory effect on platelet function. Therefore, PFA evaluation and assessment of risk for transfusion are recommended prior to large surgical procedures. Tapering off the drug may be considered according to resolving depression following surgery and reduced pain levels. Of note, cytochrome P450 enzymes can be inhibited by SSRIs when concomitantly used with nonsteroidal anti-inflammatory drugs. Those concerns warrant further investigations by spine surgeons for each case to plan accordingly [9].

\section{Bone mineral density}

Life expectancy is progressively increasing; therefore, osteoporosis is witnessed in association with depression in the elderly. Dual treatment of these two diseases is crucial in order to improve the QOL and reduce both comorbidity and mortality [21]. SSRI treatment has been commonly associated with low BMD, albeit controversially in the literature.

Rauma et al. [17] conducted a study that examined the relationship between SSRI treatment and BMD in men. They showed a negative correlation between BMD and SSRI treatment only in lower-weight men, depending on the bone site. The use of antidepressants, as indicated by recurrent MDD, was associated with lower spine BMD 
$(B=-0.047)[17,21]$.

Cortisol and catecholamine release, induced by chronic stress, may be responsible for bone loss in patients with depression. Recurrent MDD was associated with lower BMD scores for the forearm (6.5\%) and total body (2.5\%) compared with men without MDD records. Moreover, lower spine BMD was noted with recurrent MDD (4\% lower). Although the line between MDD and antidepressant treatment was unclear, both were deemed independent risk factors for osteoporosis [17].

By contrast, Saraykar et al. [21] in 2018 reported no significant difference in BMD between patients taking SSRI and those not at the spine level $(p=0.275)$ among elderly women, despite the existing trend for a reduction in BMD at the spine level. This reduction was important only when patients were on both osteoporosis drugs and SSRIs. No difference in T-scores were observed at the spine level $(p=0.393)$ [21].

Although each is an independent risk factor for reduced BMD, the treatment of osteoporosis and depression concomitantly requires careful management to avoid induced bone fragility. Therefore, regular monitoring of BMD in long-term treatment is recommended [17,21].

\section{Coexisting psychiatric conditions}

Psychiatric conditions, including anxiety and depression, are prevalent in spine patients as a result of chronic pain, disability, and social dependence. Certain subgroups, for example patients with SCI, are particularly prone to depressive symptoms. It is reported that $25 \%-30 \%$ of patients with SCI suffer from MDD [5].

Venlafaxine XR treatment for patients with SCI $(<65$ years old) presenting with MDD was examined in two studies in 2015. Fann et al. [12] reported a significant improvement in depressive symptoms by using the Maier subscale in a venlafaxine-treated group compared with a placebo group (1.6 points, $p=0.02$ ). However, in the study group, no statistically significant difference in the Hamilton Depression Rating Scale (HAM-D) 17 score was observed $(p=0.42$ ). The Maier subscale was considered to be more sensitive to changes than the HAM-D 17 score, as the former comprises six core items precluding somatic symptoms $[12,30,31]$. The venlafaxine group also showed significant improvement in SCI-related disability, according to the Sheehan Disability Scale, at 12 weeks $(p=0.005)$ [12]. Another study conducted by Richards et al. [4] evaluated nociceptive and neuropathic pain separately with the HAM-D score and Generalized Anxiety Disorder Scale. In patients with neuropathic pain, both scales indicated no significant improvements ( $p=0.815)$. By contrast, venlafaxine XR led to a statistically significant reduction in patients with nociceptive pain $(p=0.001)$ [4]. The drug was well-tolerated by the majority of the patients; however, side effects of blurred vision for the study group and constipation for the placebo group were noted in the two aforementioned studies to be $p=0.005$ and $p=0.02$, respectively $[4,12]$.

\section{Cost of spine health care}

As spinal surgeries are not uncommon, evaluation of the factors affecting costs is crucial. The mutual association between pain and depression is one of the main causes of poor surgical outcomes and increased cost. A retrospective study performed by Walid and Zaytseva [18] investigated the correlation between antidepressant use and length of hospital stay in terms of cost. Of 578 patients who underwent three common procedures, lumbar microdiscectomy, ACDF, and lumbar decompression and fusion, $24.5 \%$ were on antidepressant medication. Depression, which had been determined by antidepressant drug use, was associated with prolonged hospital stays and increased hospital charges, particularly in the lumbar decompression and fusion group $(p<0.005)$. For the lumbar microdiscectomy and ACDF groups, no statistically significant difference was detected. Patients who received antidepressants stayed 1.73 days longer in the hospital and the costs were $14.7 \%$ higher than patients not taking antidepressant medication [18].

Another study conducted by Sayadipour et al. [20] in 2016 evaluated 142 patients who underwent elective spine surgery; 41 of the 142 patients were under chronic antidepressant use. Compared with the control group, the total charges were $36 \%$ higher, the costs were $22 \%$ higher, and the fixed cost was 19\% higher among the patients taking antidepressants, who also showed a trend toward a prolonged stay in the hospital. However, none of these factors was statistically significant [20]. Prior to 2010, Sayadipour et al. [9] evaluated the relationship between intraoperative bleeding and antidepressant treatment, concluding that the mean length of hospital stay was approximately $33.3 \%$ higher in patients taking antidepressants than in those not $(p=0.0001)$. 


\section{Conclusions}

Antidepressants are used for various purposes in the field of spine surgery: (1) Antidepressants, including amitriptyline and duloxetine, are among the first line drugs to treat neuropathic pain, which is common in patients with degenerative diseases of the spine. Venlafaxine can be used for cases with SCI-related disabilities presenting with depression or nociceptive pain. (2) Preoperative treatment of depression should be considered in patients with intractable spine disease as it may improve patients satisfaction postoperatively by ameliorating postoperative pain and reducing risk of postsurgical delirium and, thus, improving overall QOL.

By contrast, antidepressants may have disadvantages: (1) They may result in prolonged hospital stay and increased cost. (2) SSRIs may increase the risk of intraoperative bleeding up to 2.5-fold and allogenic blood transfusion by 2 -fold. Bupropion may also be involved. (3) PFA is warranted preoperatively for major procedures to avoid unexpected significant intraoperative bleeding in patients taking serotonergic antidepressants. (4) The impact of antidepressants on BMD remains controversial; therefore, strict monitoring of BMD in elderly and high-risk group patients is recommended.

\section{Conflict of Interest}

No potential conflict of interest relevant to this article was reported.

\section{Author Contributions}

$\mathrm{AB}$ formulated the research idea and main objectives. $\mathrm{AB}$ and $\mathrm{CK}$ started the project by collection of data and reviewing the literature. $\mathrm{AB}$ and $\mathrm{SB}$ designed the study and determined the inclusion and exclusion criteria. CK drafted the tables and created the PRISMA figure under the supervision of $\mathrm{AB}$. OI and $\mathrm{AB}$ drafted the main bulk of the manuscript. SB and ZT critically reviewed the manuscript and participated in writing and editing the final draft. ZT is the senior supervisor of the project at all steps. $\mathrm{AB}$ and $\mathrm{OI}$ interpreted the collected data and reached the conclusion. $\mathrm{AB}, \mathrm{OI}, \mathrm{CK}, \mathrm{SB}$, and $\mathrm{ZT}$ approved the final project and held responsible for the entire project under the leadership of $\mathrm{AB}$ as a first and corresponding author.

\section{References}

1. Mercier A, Auger-Aubin I, Lebeau JP, et al. Evidence of prescription of antidepressants for non-psychiatric conditions in primary care: an analysis of guidelines and systematic reviews. BMC Fam Pract 2013;14:55.

2. Bajwa SJ, Haldar R. Pain management following spinal surgeries: an appraisal of the available options. J Craniovertebr Junction Spine 2015;6:105-10.

3. Aalto TJ, Malmivaara A, Kovacs F, et al. Preoperative predictors for postoperative clinical outcome in lumbar spinal stenosis: systematic review. Spine (Phila Pa 1976) 2006;31:E648-63.

4. Richards JS, Bombardier CH, Wilson CS, et al. Efficacy of venlafaxine XR for the treatment of pain in patients with spinal cord injury and major depression: a randomized, controlled trial. Arch Phys Med Rehabil 2015;96:680-9.

5. Craig A, Tran Y, Middleton J. Psychological morbidity and spinal cord injury: a systematic review. Spinal Cord 2009;47:108-14.

6. Elsamadicy AA, Adogwa O, Cheng J, Bagley C. Pretreatment of depression before cervical spine surgery improves patients' perception of postoperative health status: a retrospective, single institutional experience. World Neurosurg 2016;87:214-9.

7. Elsamadicy AA, Adogwa O, Lydon E, et al. Depression as an independent predictor of postoperative delirium in spine deformity patients undergoing elective spine surgery. J Neurosurg Spine 2017;27:209-14.

8. Schadler P, Shue J, Moawad M, et al. Serotonergic antidepressants are associated with increased blood loss and risk for transfusion in single-level lumbar fusion surgery. Asian Spine J 2017;11:601-9.

9. Sayadipour A, Mago R, Kepler CK, et al. Antidepressants and the risk of abnormal bleeding during spinal surgery: a case-control study. Eur Spine J 2012;21:2070-8.

10. Cardenas DD, Warms CA, Turner JA, Marshall H, Brooke MM, Loeser JD. Efficacy of amitriptyline for relief of pain in spinal cord injury: results of a randomized controlled trial. Pain 2002;96:365-73.

11. Konno S, Oda N, Ochiai T, Alev L. Randomized, double-blind, placebo-controlled phase III trial of duloxetine monotherapy in Japanese patients with chronic low back pain. Spine (Phila Pa 1976) 2016;41:1709-17. 
12. Fann JR, Bombardier CH, Richards JS, et al. Venlafaxine extended-release for depression following spinal cord injury: a randomized clinical trial. JAMA Psychiatry 2015;72:247-58.

13. Farrokhi MR, Lotfi M, Masoudi MS, Gholami M. Effects of methylene blue on postoperative low-back pain and functional outcomes after lumbar open discectomy: a triple-blind, randomized placebocontrolled trial. J Neurosurg Spine 2016;24:7-15.

14. Kumar K, North R, Taylor R, et al. Spinal cord stimulation vs. conventional medical management: a prospective, randomized, controlled, multicenter study of patients with failed back surgery syndrome (PROCESS Study). Neuromodulation 2005;8:213-8.

15. Thomson S, Jacques L. Demographic characteristics of patients with severe neuropathic pain secondary to failed back surgery syndrome. Pain Pract 2009;9:20615.

16. Farrokhi MR, Yazdanpanah H, Gholami M, Farrokhi F, Mesbahi AR. Pain and functional improvement effects of methylene blue injection on the soft tissue around fusion site after traumatic thoracolumbar fixation: a double-blind, randomized placebo-controlled study. Clin Neurol Neurosurg 2016;150:6-12.

17. Rauma PH, Pasco JA, Berk M, et al. The association between major depressive disorder, use of antidepressants and bone mineral density (BMD) in men. J Musculoskelet Neuronal Interact 2015;15:177-85.

18. Walid MS, Zaytseva NV. Prevalence of mood-altering and opioid medication use among spine surgery candidates and relationship with hospital cost. J Clin Neurosci 2010;17:597-600.

19. Robertson KL, Marshman LA. Gabapentin superadded to a pre-existent regime containing amytriptyline for chronic sciatica. Pain Med 2016;17:2095-9.

20. Sayadipour A, Kepler CK, Mago R, et al. Economic effects of anti-depressant usage on elective lumbar fusion surgery. Arch Bone Jt Surg 2016;4:231-5.

21. Saraykar S, John V, Cao B, Hnatow M, Ambrose CG, Rianon N. Association of selective serotonin reuptake inhibitors and bone mineral density in elderly women. J Clin Densitom 2018;21:193-9.
22. Sajan F, Conte JV, Tamargo RJ, Riley LH, Rock P, Faraday N. Association of selective serotonin reuptake inhibitors with transfusion in surgical patients. Anesth Analg 2016;123:21-8.

23. Bayoumi AB, Efe IE, Ozturk OC, et al. Antidepressant prescriptions in neurosurgical practice: a survey of current trends. Turk Neurosurg 2019;29:289-96.

24. Masedo AI, Hanley M, Jensen MP, Ehde D, Cardenas DD. Reliability and validity of a self-report FIM (FIM-SR) in persons with amputation or spinal cord injury and chronic pain. Am J Phys Med Rehabil 2005;84:167-76.

25. Teasell RW, Mehta S, Aubut JA, et al. A systematic review of pharmacologic treatments of pain after spinal cord injury. Arch Phys Med Rehabil 2010;91:816-31.

26. Attal N, Mazaltarine G, Perrouin-Verbe B, Albert T; SOFMER French Society for Physical Medicine and Rehabilitation. Chronic neuropathic pain management in spinal cord injury patients: what is the efficacy of pharmacological treatments with a general mode of administration? (oral, transdermal, intravenous). Ann Phys Rehabil Med 2009;52:124-41.

27. Vranken JH. Elucidation of pathophysiology and treatment of neuropathic pain. Cent Nerv Syst Agents Med Chem 2012;12:304-14.

28. Van Haelst IM, Egberts TC, Doodeman HJ, et al. Use of serotonergic antidepressants and bleeding risk in orthopedic patients. Anesthesiology 2010;112:631-6.

29. Jeong BO, Kim SW, Kim SY, Kim JM, Shin IS, Yoon JS. Use of serotonergic antidepressants and bleeding risk in patients undergoing surgery. Psychosomatics 2014;55:213-20.

30. Faries D, Herrera J, Rayamajhi J, DeBrota D, Demitrack M, Potter WZ. The responsiveness of the Hamilton Depression Rating Scale. J Psychiatr Res 2000;34:3-10.

31. Entsuah R, Shaffer M, Zhang J. A critical examination of the sensitivity of unidimensional subscales derived from the Hamilton Depression Rating Scale to antidepressant drug effects. J Psychiatr Res 2002;36:43748. 